\title{
THE BENEFITS VS POTENTIAL SIDE EFFECTS OF POSTCHEMOEMBOLIZATION FOR HEPATOCELLULAR
}

\section{CARCINOMA}

C.A. Siloși ${ }^{1}$, M.V. Boldeanu ${ }^{2,3^{*}}$, A. Andrițoiu ${ }^{4}$, M. Cojocaru ${ }^{5}$, L. Boldeanu ${ }^{6}$, C. Avramescu ${ }^{6}$, I. Siloși ${ }^{2}$

\begin{abstract}
It is known that, among chronic liver diseases, the most common cause of morbidity and/or mortality is hepatocellular carcinoma. Due to the fact that most patients are diagnosed with advanced disease, surgical resection is no longer possible. These patients constitute the ideal group for transarterial chemoembolization, which consists of the local infusion of a mixture of chemotherapeutic agents, which will cause the suppression of blood supply of the cancerous lesions, the induction of ischemia, followed by the necrosis of the tumor tissue, with a considerable improvement in the survival rate.
\end{abstract}

Keywords: chemoembolization, hepatocellular carcinoma, TACE.

${ }^{1}$ Department of Surgery, Faculty of Medicine, University of Medicine and Pharmacy of Craiova, Craiova, Romania

2 Department of Immunology, University of Medicine and Pharmacy of Craiova, Craiova, Romania

${ }^{3}$ Medico Science SRL - Stem Cell Bank Unit, Craiova, Romania

${ }^{4}$ Primary physician, Internal medicine, Military Emergency Hospital Craiova, Craiova, Romania

5 Department of Physiology, Faculty of Medicine, "Titu Maiorescu" University, Bucharest, Romania

6 Department of Microbiology, University of Medicine and Pharmacy of Craiova, Craiova, Romania

"Corresponding author: Mihail Virgil Boldeanu (boldeanumihailvirgil@yahoo.com)

Published online: 2 September 2020

\section{Introduction}

Hepatocellular carcinoma (HCC) is a common cause of cancer-related death. Most of this cancer burden falls on developing countries, with the highest incidence (1). Prolonged alcohol use and nonalcoholic steatohepatitis (NASH) are also significant risk factor (2). The incidence of $\mathrm{HCC}$ is growing worldwide globally, HCC is an even greater public health concern, as it is the third leading cause of cancer-related deaths worldwide (3). It occurs most often in the setting of cirrhosis, usually related to chronic hepatitis $C$ virus (HCV) infection or chronic hepatitis $B$ virus (HBV) infection $(1,4)$. The treatment of solid tumours, including HCC, still remains one of the major unsolved problem. Over the last decade, the management of solid tumors has expanded and now includes expanded small molecular drugs used in targeted therapy, monoclonal antibodies and immune checkpoint inhibitors used in 
immunotherapy, modern technology used surgery (5-8).

Despite the numerous existing strategies to treat HCC, the 5-year survival rate remains below $12 \%$ (1). In developing nations, survival rates are as low as $5 \%$ (3). Surgical resection, transplantation and ablation are potentially curative treatment options for HCC (9). Unfortunately, only a minority of patients are eligible for these treatments at the time of diagnosis (2).

In contrast, patients frequently have symptoms of cancer and liver failure, unless their tumors are identified early by surveillance methods (10).

Hepatocellular carcinoma (HCC) is the most common type of primary liver cancer. This type of tumor increases a large number of blood vessels. These blood vessels get the largest amount of blood from the hepatic artery, while the rest of the liver tissue receives blood from the portal vein. Because of this, doctors can block the hepatic artery to reduce the blood supply to the tumor, without affecting the rest of the liver. Embolization is a treatment that blocks or slows blood supply to tissues or an organ. It can be used to block the flow of blood to a tumor so that the cancer cells die. When the material used to block blood supply also delivers tumor chemotherapy drugs, this is called chemoembolization (4, 10).

\section{Transarterial} chemoembolization (TACE)

Transarterial chemoembolization (TACE) is a specific type of chemoembolization that blocks the hepatic artery for the treatment of liver cancer. For patients with more advanced disease, several treatments have been developed to slow the progression of the disease. These include many livertargeted therapies, such as mild transarterial embolization (TAE), conventional transarterial chemoembolization (CTACE), pearls eluting TACE drugs (DEB-TACE), and lithium-90 radioembolization (90Y) $(11,12)$.

The liver is unique because it has two blood supplies - an artery (liver artery) and a large vein (portal vein). The normal liver receives about 75 percent of the blood supply through the portal vein and only 25 percent through the hepatic artery. But when a tumor grows in the liver, it receives almost all of the blood from the hepatic artery. Chemotherapy drugs injected into the hepatic artery blood is no longer provided by the tumor, while the liver continues to be supplied by blood from the portal vein. This also allows a higher concentration of anti-cancer drugs to be in contact with the tumor for a longer period of time. However, at the time of writing, CTACE is the only liver-targeted method that has been shown to deliver survival benefit in randomized controlled trials (13, 14). 
TACE attacks cancer in two ways. First, it offers a very high concentration of chemotherapy or anti-cancer drugs, directly in the tumor, without exposing the whole body to the effects of these drugs. Secondly, the procedure interrupts the blood supply to the tumor, taking the anticancer drugs on the spot and lacking the tumor of oxygen and nutrients it needs to grow.

As such, cTACE is currently the standard of care for patients who meet the criteria for intermediate-stage HCC, as defined by the Barcelona Clinical Liver Cancer (BCLC) guidelines (15).

The ability to assess the response to treatment after TACE is essential to determine the effectiveness of previous treatments and the need for withdrawal. The imaging response to treatment also has the potential to improve patient selection and predict patient outcomes (16).

However, traditional imaging criteria, such as the World Health Organization (WHO) and Response Evaluation Criteria in Solid Tumors (RECIST), focus on tumor size as a marker of treatment $(17,18)$. This is problematic in HCC, as size-based criteria have been shown to be poor predictors of patient survival following cytostatic treatment methods, such as TACE (19). It is important that clinicians treating patients with HCC are familiar with these concepts.

Unlike most malignancies, the diagnosis of HCC can be made only on imaging, without the need for pathological confirmation (20). This requires imaging centers to pay close attention to the highest standards during imaging acquisition and for radiologists to follow the protocols defined during image interpretation and reporting.

Newer imaging technologies, such as volumetric analysis, dual energy CT, conical beam CT, and perfusion CT can offer additional benefits for patients with HCC (21).

Treatment of HCC depends on the stage of the disease. The intermediate stage HCC comprises the largest subgroup of patients with the disease and is characterized by a substantial heterogeneity. The standard therapeutic approach, TACE, is probably overused and may not be suitable for all patients with intermediate-stage HCC. In patients with large tumors, multi-nodular spread or impaired liver function, TACE may not be optimal and other treatments may be considered a first-line treatment. These include surgery, percutaneous ablation, radioembolization or systemic treatment. In addition, patients who do not achieve complete or partial necrosis (TACE insufficiency) and patients with early recurrence after TACE should be managed individually, taking into account the systemic treatments usually reserved for advanced disease (22). 


\section{TACE techniques}

Transarterial chemoembolization is a nonsurgical way of treating certain tumors in the liver. TACE places chemotherapy and synthetic materials called embolic agents in a blood vessel that feeds a cancerous tumor to cut off the tumor's blood source and capture chemotherapy inside the tumor. TACE delivers drugs directly to the liver tumor using a catheter (a small flexible tube) that is inserted into an artery in the groin area.

The TACE procedure is performed by a specialist, usually an interventional radiologist, who makes a very small incision in the groin to insert the catheter into the femoral artery. The catheter is then directed through the aorta and into the hepatic artery, which supplies the main blood supply to a liver tumor.

TACE can be used to provide both chemotherapy and a substance that leads to coagulation, or embolization. Embolization results in the blockage of blood supply to the tumor, which causes cancer cells to die from lack of oxygen and nutrients.

The substance used may be either tiny "microspheres", metal coils, or a specialized gel. Chemotherapy is delivered directly to the cancer at higher concentrations than can be achieved by its administration through a vein. Loss of blood flow from embolization prevents chemotherapy from being washed away from cancer, making it more effective (23).
The reason for conventional TACE is that the intra-arterial injection of a viscous emulsion, made by a chemotherapeutic drug, such as doxorubicin or cisplatin mixed with iodized oil, followed by embolization of the blood vessel with particles of gelatin sponge or other embolic agents resulted in a potent intensified cytotoxic effect of ischemia (24). An important limitation of conventional TACE was the inconsistency in the technique and treatment schedules. This limitation prevented the acceptance of TACE as a standard oncological treatment.

TACE drug-using beads (DEB-TACE) offer levels of consistency and repeatability that are not available with conventional TACE and offer the possibility of implementing a more standardized approach to HCC treatment (25).

In recent years, calibrated synthetic microspheres have been developed that are loaded with the chemotherapeutic agent. These ensure a more uniform and prolonged release of the drug and reach high concentrations of the chemotherapeutic agent in tumor cells, reducing its passage through the systemic circulation and thereby reducing side effects (26-28).

Therefore, drug-eluting microspheres (DEMs) have been introduced to ensure a more sustained and selective delivery of tumors for permanent embolization. These DEMs can load different drugs and deliver them in a sustained manner for a long time. This approach ensures the administration 
of high concentrations of chemotherapeutic agents on tumors without increasing systemic concentrations and promotes ischemia and tumor necrosis (28).

The DEB-TACE technique differentiates it from the conventional TACE, in which the chemotherapeutic agent is administered together with lipiodol and subsequently the occlusion material. In addition, the safety of both treatments was evaluated and no differences were found in their safety profiles. In addition, this method does not increase survival or decrease local recurrence (27).

Conventional transcatheter arterial chemoembolization (c-TACE) is a first-line palliative treatment widely used for patients with non-resectable HCC. Defined as CTACE in the most distal part of the subsegmental hepatic artery, it is mainly performed for HCC $\leq 5 \mathrm{~cm}(29,30)$. TACE is considered a therapeutic option. It is used mainly in hepatocellular or colorectal carcinoma, neuroendocrine or melanoma metastases (31). Despite the effectiveness of c-TACE, to date, the planning of the technique and procedures has not yet been standardized (28).

\section{Indications and contraindications of} TACE

TACE can be performed in patients with tumors that have developed from the liver itself (hepatocellular carcinoma) or sometimes to patients with tumors from other parts of the body, such as colon cancer, which have spread to the liver, if not there are too many spreading sites (metastases), of which the most common are acute cholecystitis and leukopenia. Other complications described are pulmonary thromboembolism, liver ischemia, liver abscess, bile duct lesions and, less frequently, acute pancreatitis (3235).

TACE is most beneficial for patients whose disease is predominantly confined to the liver, regardless of whether the tumor started in the liver or spread to the liver (metastasis) from another organ. Cancers that can be treated by TACE include : hepatoma or hepatocellular carcinoma (primary liver cancer), cholangiocarcinoma (primary cancer of the bile ducts in the liver), metastasis (spread) to the liver from (colon cancer, breast cancer, carcinoid tumors and other neuroendocrine tumors, islet cell tumors of the pancreas, ocular melanoma, sarcoma, other vascular primary tumors in the body).

Depending on the number and type of tumors, TACE may be used as the sole treatment or may be combined with other treatment options, such as surgery, chemotherapy, radiotherapy or ablation.

Recommendations for the use of TACE in Patients with Hepatocellular Carcinoma as reported in the Clinical Practice Guidelines of European Association for the Study of the Liver and European Organization for Research and Treatment of Cancer (EASL-EORTC) (29): 
- TACE is recommended for patients with intermediate-stage HCC (multinodular asymptomatic tumors without vascular invasion or extrahepatic spread) (grade of recommendation $1 \mathrm{~A}$, GR: 1A)

- TACE is discouraged in patients with decompensated liver disease, advanced liver dysfunction, macroscopic invasion, or extrahepatic spread (GR: 1B)

- The use of drug-eluting beads has shown similar response rates than Gelfoam-lipiodol particles associated with less systemic adverse events (GR: 2B)

- Selective intra-arterial chemotherapy, bland embolization, and lipiodolization are not recommended for the management of HCC (GR: 2B)

Absolute and Relative Contraindications for Conventional Transcatheter Arterial Chemoembolization in Patients with Hepatocellular Carcinoma (22):

Absolute contraindications:

- Technical contraindications to hepatic intra-arterial treatment (e.g., untreatable arterio-venous fistula);

- Renal insufficiency (creatinine $\geq 2$ $\mathrm{mg} / \mathrm{dL}$ or creatinine clearance $\leq 30$ $\mathrm{mL} / \mathrm{min})$;

- Severely reduced portal vein flow;

- Decompensated cirrhosis (ChildPugh B 8 or higher) including: jaundice, clinical encephalopathy, hepatorenal syndrome;

- Extensive tumor with massive replacement of both lobes.
Relative contraindications (Adapted from Raoul et al. (22)):

- Comorbidities involving compromised organ function: active cardiovascular disease, or active lung disease;

- Untreated varices at high risk of bleeding;

- $\quad$ Tumor size $\geq 10 \mathrm{~cm}$;

- Bile duct occlusion or incompetent papilla due to stent or surgery.

\section{The benefits of TACE}

Transarterial chemoembolization is most often used to treat liver cancer, but it can also be used in patients whose cancer has spread to other areas of the body. It can also be used as adjuvant treatment before or after surgical resection or as bridge therapy before liver transplant.

The palliative effect in nonresectable HCC using TACE allows local tumor control in 15 to $60 \%$ of cases and 5-year survival rates ranging from $8-43 \%$. The potential curative treatment option allows local tumor control from 18 to $63 \%$. The neoadjuvant treatment option of TACE in combination with other treatment options such as percutaneous ethanol injection (PEI) or radiofrequency ablation (RFA) reach local tumor control values between $80-96 \%$. The effect of reducing TACE before liver transplantation reaches 5-year survival rates of $59-93 \%$. The symptomatic treatment option of TACE is used to counteract the pain directly caused by HCC 
and acute / subacute bleeding in HCC. The local tumor response reaches $88 \%$ and the bleeding control is 83 to $100 \%$ (36).

Chemotherapy is delivered directly to the cancer at higher concentrations than can be achieved by its administration through a vein. Loss of blood flow from embolization prevents chemotherapy from being washed away from cancer, making it more effective (23).

\section{Potential side effects of TACE}

Even though TACE is considered a safe procedure, it is not without complications. There is a small risk of bleeding and/or deterioration of blood vessels, as well as infection. Intravenous contrast is also used to target the catheter, which may be at risk for people with kidney disease. This is associated with a low risk of allergic reaction, kidney injury or hypothyroidism (reduced function of the thyroid gland). After TACE, the treated tumors will often significantly decrease or even die, without remaining cancerous cells alive (23).

Because TACE usually causes significant cancer cell death immediately after the procedure, it can lead to a lot of inflammation in the area. Patients who typically undergo TACE (approximately $60 \%-80 \%$ of the time) have postembolization syndrome of abdominal pain, fever and low energy within a few days after the procedure. Also, some liver function may have blood test results become abnormal. This syndrome is usually resolved over time with pain medications and sometimes intravenous fluids if needed. Antibiotics are not routinely required (23).

Chan $\mathrm{AO}$ et al., noted that side effects and complications after TACE range from fever, upper abdominal pain and vomiting to acute or chronic liver cell failure (37).

Marcacuzco Quinto A et al., asserts that transarterial chemoembolization is considered a therapeutic option. It is used mainly in hepatocellular carcinoma or colorectal metastases, neuroendocrine or hepatic melanoma. Although it is considered a safe procedure, TACE has complications, such as acute cholecystitis, which is the most common. Other complications related to the procedure include pulmonary embolism, liver abscess, bile duct injury, gastric mucosal injury and, less commonly, acute pancreatitis (34). Marcacuzco Quinto A et al., in 2018, found the following incidents for complications of TACE in the treatment of liver tumors (34) (Table 1 adapted).

CTCAE represents the Common Criteria for Terminology for Adverse Events; these criteria are also referred to as "common toxicity criteria" (35). In CTCAE, an adverse event $(A E)$ is defined as any abnormal clinical finding temporally associated with the use of cancer therapy; no causality is necessary. These criteria are used to manage the administration and dosage of chemotherapy and in clinical trials to ensure standardization and consistency in defining treatment-related toxicity. 
Chan $\mathrm{AO}$ et al., identified factors that seem to predispose patients to the development of irreversible acute hepatic decompensation after TACE, namely high dose of cisplatin, high basal bilirubin levels, prolonged prothrombin time and advanced cirrhosis. Despite the high percentage of patients who developed hepatic decompensation after TACE, irreversible damage occurred only in a minority (37).

\begin{tabular}{|c|c|c|c|c|}
\hline Parameters & $\begin{array}{c}\text { Total } \\
(n=322)\end{array}$ & $\begin{array}{c}\text { HCC } \\
(n=315)\end{array}$ & $\begin{array}{l}\text { ICC } \\
(n=2)\end{array}$ & $\begin{array}{c}\text { Hepatic } \\
\text { metastasis } \\
(n=5)\end{array}$ \\
\hline \multicolumn{5}{|l|}{ Major complications } \\
\hline AD & $6(1.8 \%)$ & 6 & 0 & 0 \\
\hline Acute cholecystitis & $4(1.2 \%)$ & 3 & 0 & 1 \\
\hline Acute pancreatitis & $3(0.9 \%)$ & 3 & 0 & 0 \\
\hline Liver abscess & $1(0.3 \%)$ & 0 & 1 & 0 \\
\hline Liver rupture & $1(0.3 \%)$ & 1 & 0 & 0 \\
\hline Renal function decline & $1(0.3 \%)$ & 1 & 0 & 0 \\
\hline \multicolumn{5}{|l|}{ Minor complications } \\
\hline PES & $71(22 \%)$ & 67 & 1 & 3 \\
\hline Abdominal pain & $64(20 \%)$ & 58 & 2 & 4 \\
\hline Fever & $60(19 \%)$ & 55 & 2 & 3 \\
\hline Inguinal hematoma & $17(5 \%)$ & 16 & 1 & 0 \\
\hline Liver function decline & $16(5 \%)$ & 16 & 0 & 0 \\
\hline \multicolumn{5}{|c|}{ Adverse effects according to CTCAE } \\
\hline Grade 1 & $231(71.7 \%)$ & 229 & 0 & \\
\hline Grade 2 & $36(11.2 \%)$ & 36 & 0 & \\
\hline
\end{tabular}

Table 1. Incidence of complications and adverse effects associated with transarterial chemoembolization. ICC: intrahepatic cholangiocarcinoma; HCC: hepatocellular carcinoma; CTCAE: Common Terminology Criteria for Adverse Events (The National Cancer Institute); AD: ascitic decompensation; PES: post-embolization syndrome

Dhamija $E$ et al., in 2015, found that biliary complications following TACE are rare. The diagnosis should be clinically suspected and confirmed by imaging. Treatment depends on the severity. The application of specific measures can minimize its frequency (38).

In an assessment of their own case law and specialty literature, Tasneem $A$ et al. (2013) found that the potential predictors of complications of liver tumors after chemoembolization, the following parameters: stage hepatic disease, chemoembolization dose, tumor size, portal vein thrombosis, hepatic decompensation. Transarterial chemoembolization, although generally safe, can lead to serious complications in patients with advanced liver disease (31).

The results of an extensive literature review in the treatment of $\mathrm{HCC}$ unresectable with TACE were combined with our own clinical experience to identify 
factors that could predict survival after TACE.

Andrițoiu A et al. (39) consider that the minimal-invasive approach of post-tace hepatic abscess represents an effective solution, in which the aspirative puncture and percutaneous drainage associated with pharmacological support can lead to the therapeutic resolution of this complication in the vast majority of cases.

\section{Conclusions}

TACE is used to treat a liver cancer that cannot be treated with surgery, or by other treatments that try to cure it. Transarterial chemoembolisation, is a palliative treatment, which means it is not going to cure your cancer and is used to control tumour growth and try to shrink the tumour.

TACE, though generally safe, may lead to serious complications in patients with advanced liver disease. The common complications of TACE are postembolization syndrome (fever, abdominal pain, nausea, vomiting), impaired liver function, and leukocytopenia. Post-procedure syndrome was associated with increased tumour size and lipoidol dose.

\section{Conflict of interest}

The authors declare no conflict of interest.

\section{References}

1. El Serag HB. Hepatocellular carcinoma: an epidemiologic view. J Clin Gastroenterol, 2002;35(5 Suppl2):S72-8. https://doi.org/10.1097/00004836-200211002-00002

2. Singal AG, El-Serag HB. Hepatocellular carcinoma from epidemiology to prevention: translating knowledge into practice. Clin Gastroenterol Hepatol 2015;13(12):2140-2151. https://doi.org/10.1016/i.cgh.2015.08.014

3. Bosetti C, Turati F, La Vecchia C. Hepatocellular carcinoma epidemiology. Best Pract Res Clin Gastroenterol,2014;28(5):753-770. https://doi.org/10.1016/i.bpg.2014.08.007

4. Kanwal F, Hoang T, Kramer JR, Asch SM, Goetz MB, Zeringue A, Richardson P, El-Serag HB. Increasing prevalence of $\mathrm{HCC}$ and cirrhosis in patients with chronic hepatitis C virus infection. Gastroenterology, 2011;140(4):1182-1188. https://doi.org/10.1053/i.gastro.2010.12.032

5. Marrero JA, Kulik LM, Sirlin CB, Zhu AX, Finn RS, Abecassis MM, Roberts LR, Heimbach JK. Diagnosis, Staging, and Management of Hepatocellular Carcinoma: 2018 Practice Guidance by the American Association for the Study of Liver Diseases. Hepatology, 2018 Aug; 68(2):723-750. https://doi.org/10.1002/hep.29913

6. SA Artene, C Tuţă, Alexandra Dragoi, Oana Alexandru, Purcaru Stefana Oana, Daniela Elise Tache, Maria Mihaela Dănciulescu, MV Boldeanu, CA Siloşi, Anica Dricu. Current and emerging EGFR therapies for glioblastoma. J Immunoassay Immunochem.2018;39(1):1-11.

https://doi.org/10.1080/15321819.2017.1411816

7. Artene SA, Turcu-Stiolica A, Ciurea ME, Folcuti C, Tataranu LG, Alexandru O, Purcaru OS, Tache DE, Boldeanu MV, Silosi C, Dricu A. Comparative effect of immunotherapy and standard therapy in patients with high grade glioma: a meta-analysis of published clinical trials. Sci Rep. 2018 Aug 7;8(1):11800. doi: 10.1038/s41598-018-30296-x. https://doi.org/10.1038/s41598-018-30296-x

8. Chihua Fang, Peng Zhang and Xiaolong Qi. Digital and intelligent liver surgery in the new era: Prospects and dilemmas. EBioMedicine. 2019 Mar; 41: 693-701. https://doi.org/10.1016/i.ebiom.2019.02.017

9. Forner A, Llovet JM, Bruix J. Hepatocellular carcinoma. Lancet, 2012; 379(9822):1245-55. https://doi.org/10.1016/S0140-6736(11)61347-0 
10. Bruix J, Reig M, Sherman M. Evidence-based diagnosis, staging, and treatment of patients with hepatocellular carcinoma. Gastroenterology, 2016;150(4):835-53.

https://doi.org/10.1053/i.gastro.2015.12.041

11. Pesapane F, Nezami N, Patella F, Geschwind JF. New concepts in embolotherapy of HCC. Med Oncol, 2017;34:58.

https://doi.org/10.1007/s12032-017-0917-2

12. Molvar C, Lewandowski RJ. Intra-arterial therapies for liver masses: data distilled. Radiologic Clinics of North America,2015;53(5):973-984. https://doi.org/10.1016/j.rcl.2015.05.011

13. Llovet JM, Real MI, Montaña X, Planas R, Coll S, Aponte J. Arterial embolisation or chemoembolisation versus symptomatic treatment in patients with unresectable hepatocellular carcinoma: a randomised controlled trial. Lancet, 2002;359(9319): 1734-9. https://doi.org/10.1016/S0140-6736(02)08649-X

14. Cammà $C$, Schepis $F$, Orlando A, Albanese M, Shahied L, Trevisani F. Transarterial chemoembolization for unresectable hepatocellular carcinoma: meta-analysis of randomized controlled trials. Radiology, 2002;224(1):47-54. https://doi.org/10.1148/radiol.2241011262

15. EASL-EORTC clinical practice guidelines: management of hepatocellular carcinoma $\mathrm{J}$ Hepatol, 2012;56(4):908-43. https://doi.org/10.1016/j.jhep.2011.12.001

16. Sala M, Llovet JM, Vilana R, Bianchi L, Solé M, Ayuso C. Initial response to percutaneous ablation predicts survival in patients with hepatocellular carcinoma. Hepatology,2004;40(6):1352-60. https://doi.org/10.1002/hep.20465

17. Miller $A B$, Hoogstraten $B$, Staquet $M$, Winkler $A$. Reporting results of cancer treatment. Cancer, 1981;47(1):207-14.

https://doi.org/10.1002/10970142(19810101)47:1<207::AIDCNCR2820470134>3.0.CO;2-6

18. Therasse P, Arbuck SG, Eisenhauer EA, Wanders J, Kaplan RS, Rubinstein L. New guidelines to evaluate the response to treatment in solid tumors. European Organization for Research and Treatment of Cancer, National Cancer Institute of the United States, National Cancer Institute of Canada. J Nat Cancer Inst, 2000;92(3):205-216. https://doi.org/10.1093/inci/92.3.205

19. Shepherd FA, Rodrigues Pereira J, Ciuleanu T, Tan EH, Hirsh V, Thongprasert S. Erlotinib in previously treated non-small-cell lung cancer. $\mathrm{N}$ Engl $\mathrm{J}$ Med,
2005;353(2):123-32.

https://doi.org/10.1056/NEJMoa050753

20. Leoni S, Piscaglia F, Golfieri R, Camaggi V, Vidili G, Pini $P$. The impact of vascular and nonvascular findings on the noninvasive diagnosis of small hepatocellular carcinoma based on the EASL and AASLD criteria. Am J Gastroenterol, 2010;105(3):599609.

https://doi.org/10.1038/ajg.2009.654

21. Vande Lune, Abdel Aal AK, Klimkowski S, Zarzour JG, Gunn AJ. Hepatocellular Carcinoma: Diagnosis, Treatment Algorithms, and Imaging Appearance after Transarterial Chemoembolization. J Clin Transl Hepatol,2018;6(2):175-188. https://doi.org/10.14218/JCTH.2017.00045

22. Raoul JL1, Sangro B, Forner A, Mazzaferro V, Piscaglia F, Bolondi L, Lencioni R. Evolving strategies for the management of intermediate-stage hepatocellular carcinoma: available evidence and expert opinion on the use of transarterial chemoembolization. Cancer Treat Rev, 2011;37(3):212-20. https://doi.org/10.1016/..ctrv.2010.07.006

23. West HJ, Jin JO. Transarterial Chemoembolization. JAMA Oncol, 2015;1(8):1178.

https://doi.org/10.1001/jamaoncol.2015.3702

24. Lencioni R, de Baere $T$, Burrel M. et al. Transcatheter treatment of hepatocellular carcinoma with Doxorubicin-loaded DC Bead (DEBDOX): technical recommendations. Cardiovasc Intervent Radiol, 2012;35(5):980-985 https://doi.org/10.1007/s00270-011-0287-7

25. Yamaguchi A, Taniguchi $\mathrm{H}$, Kunishima $\mathrm{S}$, Koh $\mathrm{T}$, Yamagishi $\mathrm{H}$. Correlation between angiographically assessed vascularity and blood flow in hepatic metastases in patients with colorectal carcinoma. Cancer,2000;89(6):1236-44. https://doi.org/10.1002/10970142(20000915)89:6<1236::AID-CNCR7>3.0.CO;2-K

26. Dhanasekaran R, Kooby DA, Staley CA, Kauh JS, Khanna V, Kim HS. Comparison of conventional transarterial chemoembolization (TACE) and chemoembolization with doxorubicin drug eluting beads (DEB) for unresectable hepatocelluar carcinoma (HCC). J Surg Oncol, 2010;101(6):476-80. https://doi.org/10.1002/jso.21522

27. Lammer J, Malagari K, Vogl T, Pilleul F, Denys A, Watkinson A, Pitton M, Sergent $G$, Pfammatter $T$, Terraz S, Benhamou Y, Avajon $\mathrm{Y}$, Gruenberger $\mathrm{T}$, Pomoni $M$, Langenberger $H$, Schuchmann $M$, Dumortier J, Mueller C, Chevallier P, Lencioni R; 
PRECISION V Investigators. Prospective randomized study of doxorubicin-eluting-bead embolization in the treatment of hepatocellular carcinoma: results of the PRECISION V study. Cardiovasc Intervent Radiol, 2010;33(1):41-52.

https://doi.org/10.1007/s00270-009-9711-7

28. Nouri YM, Kim JH, Yoon HK, Ko HK, Shin JH, Gwon DI. Update on Transarterial Chemoembolization with Drug-Eluting Microspheres for Hepatocellular Carcinoma. Korean J Radiol, 2019;20(1): 34-49. https://doi.org/10.3348/kjr.2018.0088

29. Galle PR, Tovoli F, Foerste F, Worns MA, Cucchetti A, Bolondi $L$. The treatment of intermediate stage tumours beyond TACE: From surgery to systemic therapy. J Hepatol, 2017;67(1):173-183. https://doi.org/10.1016/j.jhep.2017.03.007

30. Miyayama S. Ultraselective conventional transarterial chemoembolization: When and how? Clin Mol Hepatol, 2019, PMID: 31022779 Free Article https://doi.org/10.3350/cmh.2019.0016

31. Tasneem A., Abbas Z., Luck N., Hassan S., Faiq. S. Adverse events following transarterial chemoembolization for hepatocellular carcinoma and factors predicting such events. J Pak Med Assoc, 2013;63(2):239-44.

32. Alcívar-Vásquez JM, Ontanilla-Clavijo G, Ferrer-Ríos MT and Pascasio-Acevedo JM. Acute necrotizing pancreatitis after transarterial chemoembolization of hepatocellular carcinoma: an unusual complication. Acute necrotizing pancreatitis after transarterial chemoembolization of hepatocellular carcinoma: An unusual complication. Rev Esp Enferm Dig, 2014;106(2):147-9.

33. Kim SI, Jin YJ, Cho SG, Shin WY, Kim JM, Lee JW. Duodenal perforation and esophageal ischemia following transarterial chemoembolization for hepatocellular carcinoma: A case report. Medicine
(Baltimore), 2016;95(27): e3987. https://doi.org/10.1097/MD.0000000000003987

34. Marcacuzco Quinto A, Nutu OA, San Román Manso $R$, Justo Alonso, Calvo Pulido Manrique Municio A, García-Sesma Á, Loinaz Segurola C, Martínez Caballero J, Jiménez Romero LC. Complications of transarterial chemoembolization (TACE) in the treatment of liver tumors. Cir Esp, 2018;96(9):560-567. https://doi.org/10.1016/i.ciresp.2018.06.004

35.https://ctep.cancer.gov/protocoldevelopment/electronic applications/docs/CTCAE v5 Quick Reference $8.5 \times 1$ $\underline{1 . p d f}$

36. Vogl TJ, Zangos S, Balzer JO, Nabil M, Rao P, Eichler $\mathrm{K}$, Bechstein WO, Zeuzem S, Abdelkader A. Transarterial chemoembolization (TACE) in hepatocellular carcinoma: technique, indication and results. Rofo, 2007;179(11):1113-26. https://doi.org/10.1055/s-2007-963285

37. Annie O. Chan, Man-Fung Yuen, Chee-Kin Hui, Wai-Kuen, Ching-Lung Lai. A prospective study regarding the complications of transcatheter intraarterial lipiodol chemoembolization in patients with hepatocellular carcinoma. Cancer, 2002;94(6):174752. https://doi.org/10.1002/cncr.10407

38. Dhamija E, Paul SB, Gamanagatti SR, Acharya S. Biliary complications of arterial chemoembolization of hepatocellular carcinoma. Diagn Interv Imaging, 2015; 96(11):1169-75. https://doi.org/10.1016/i.diii.2015.06.017

39. Andritoiu A, Silosi CA, Resceanu A, Mercut D. Abces hepatic post-chemoembolizare rezolvat prin drenaj continuu percutan, în Ecografia interventionala in patologia hepato-bilio-pancreatica, sub redactia Dorin Mercut si Alexandru Andritoiu, Ed. Info, 2018, 215226. 